\title{
Spatio-temporal dependencies in functional connectivity in rodent cortical cultures
}

\author{
Matthew C. Spencer ${ }^{1 *} \quad$ Abstract \\ Julia H. Downes ${ }^{1}$, Dimitris Xydas ${ }^{1}$, \\ Mark W. Hammond ${ }^{1,2}$ \\ Victor M. Becerra ${ }^{1}$, Benjamin \\ J. Whalley ${ }^{1,2}$, Kevin Warwick ${ }^{1}$ \\ Slawomir J. Nasuto \\ Cybernetic Research Group \\ School of Systems Engineering \\ University of Reading. \\ Whiteknights, RG6 6AY, UK \\ 2 School of Pharmacy, \\ University of Reading \\ Whiteknights, RG6 6AP UK \\ Received 2011/11/10 \\ Accepted 2012/01/22 \\ Keywords \\ functional connectivity $\cdot$ cortical cultures $\cdot$ multi-electrode arrays
}

Models of functional connectivity in cortical cultures on multi-electrodes arrays may aid in understanding how cognitive pathways form and improve techniques that aim to interface with neuronal systems. To enable research on such models, this study uses both data- and model-driven approaches to determine what dependencies are present in and between functional connectivity networks derived from bursts of extracellularly recorded activity. Properties of excitation in bursts were analysed using correlative techniques to assess the degree of linear dependence and then two parallel techniques were used to assess functional connectivity. Three models presenting increasing levels of spatio-temporal dependency were used to capture the dynamics of individual functional connections and their consistencies were verified using surrogate data. By comparing network-wide properties between model generated networks and functional networks from data, complex interdependencies were revealed. This indicates the persistent co-activation of neuronal pathways in spontaneous bursts, as can be found in whole brain structures.

\section{Introduction}

Cultures of cortical neurones on Multi-Electrode Arrays (MEAs; specialised glass dishes with a lattice of embedded electrodes), are good platforms for investigating anatomical and functional neuronal networks because they are maintainable for weeks or months, can be recorded for extended periods, can be observed under a microscope, and experimental control can be maintained over the chemical and electrical environments of the neurones [1-4]. Also, these cultures are an accessible platform for studying the computational properties of biological neuronal networks to gain understanding of their behaviour and to potentially enable their use for control of robotic systems $[5,7]$.

These neurones are initially dissociated to sever synaptic connections, but upon seeding onto an MEA, they begin to reintegrate their network through the extension of neurites and the formation of synapses (governed by complex biological processes, effectively producing a random network), and they begin to exhibit action potentials within several days, leading to spontaneous and highly synchronous bursts of excitation around the 14th day in vitro (DIV 14) $[1,7]$. These bursts are reminiscent of both developmental behaviour in embryonic brains, thought to be responsible for optimising the network for information processing [8], and of the pathological behaviour observed in ailments like epilepsy or ataxia [9]. They also may be an obstacle to experimentally inducing

*E-mail: matthew.spencer@reading.ac.uk synaptic plasticity [2, 9]. Nonetheless, these bursts of activity represent a natural separation of time-scales [10] and are ideal phenomena for gaining insight into the inter-regional interactions [11] of these neuronal networks because they are formed spontaneously and tend to involve most, if not all, of the observable network.

These bursts likely represent cascades of excitation through the synaptic connections of a neuronal network [1]. While synaptic changes can occur quickly, the anatomical structure, in the absence of outside intervention, generally changes on a much slower time-scale [12]. While this relatively stable anatomical structure lends a degree of consistency to these bursts, there is also significant variation, not only in the burst intensities and locations, but also in the inter-channel synchrony [8, 13]. Some cultures tend to exhibit burst sizes drawn from a narrow, monomodal distribution (referred to as a fixed distribution), while others exhibit wider mono-modal (variable distribution) or even distinctly multimodal distributions (typically bimodal distributions) [7]. While the fixed distribution cultures produce a progression of bursts that are very similar in intensity, the other two culture types produce widely varying behaviour, which may point to distinct hierarchical arrangements of subnetworks or modules or a variety of modes of activation [14].

Techniques to measure the anatomical connectivity of live neuronal networks are limited [15], but functional connectivity (how the regions of the network interact) can be assessed by through commonalities in the spiking patterns recorded from these different regions with an MEA $[16,17]$ and can give insight into the anatomical structure when observed over long durations [12]. Within bursts of excitation, the functional connectivity of the culture can be assessed, creating functional connectivity networks (FCNs) by treating the regions of neuronal tissue 

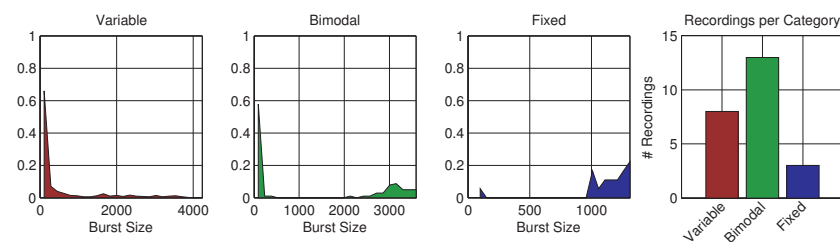

Figure 1. Burst Size Distributions. Typical distributions are shown to illustrate the three classifications: variable is wide and unimodal, bimodal has two distinct modes, and fixed is narrow and unimodal. The bar graph denotes the number of cultures of each class that were present in the analysed dataset.

surrounding each recording electrode as nodes and significantly synchronous activity between regions as edges [11]. These FCNs exhibit small-world characteristics, which are important for information flow and storage [18] and are common in self-organising systems, including brain networks [11]. Further, it is likely that information may not be only encoded in the static structures of these networks, but also in how they evolve from burst to burst [19].

Understanding the precise temporal and spatial dependencies between the regions of the neuronal networks in these bursts could lead to a better understanding of both why and when the bursts occur, as well as their physiological significance. Further, understanding these dependencies may ultimately lead to models that are capable of capturing the inter and intra burst dynamics, thus allowing classification and interpretation of network wide spiking activity and help guide intervention to access the computational capacity of these networks. To this end, this study aims to investigate the temporal and spatial dependencies in the robust and recurrent spontaneous bursts of cultures aged $\approx 21$ DIV [8].

The following paper uses data-driven and modelling techniques to investigate the importance of these spatio-temporal dependencies. Properties such as temporal dependence on prior bursts and the range dependence of synchronous links are tested using linear correlative analyses, while the importance of spatial dependencies in FCNs on their small-world properties are tested using three models that incorporate increasing spatio-temporal dependence structures. Ultimately, this study demonstrates the importance of inter-edge dependencies and the complexities in the temporal dependence between the FCNs of bursts. ${ }^{1}$

\section{Methods}

\subsection{Cortical cultures and spike detection}

The cultures were prepared from chemically and mechanically dissociated cortical neurones from foetal Wistar rats and seeded onto substrate embedded MEAs from MultiChannel Systems GmbH which provided 60 recording electrodes (59 viable recording channels and one ground), arranged in a square lattice, $200 \mu \mathrm{m}$ apart [6, 7]. Fluctuations in extracellular membrane potential were observed at these 59 sites for 20 to 40 minutes and spikes of negative polarity, representative of single-unit or multiple single-unit excitatory events [22], were identified

\footnotetext{
1 This study is based on work presented in IEEE CIS 2010 [21]
}

using a sufficiently sensitive adaptive threshold to avoid missing any relevant events. The times of these spikes and the channels on which they occurred were recorded and cleaned to remove positive spikes and other artefacts.

The cultures used for this analysis were drawn from two data sets: the publicly available data set from [7] and a data set recorded at the University of Reading. Cultures from both labs were produced using the same protocol and plated at similar densities and were found to have very similar bursting characteristics.

In choosing recordings for this study, two criteria were considered: that there be a sufficient number of network-wide bursts for statistical significance, and that the natural behaviour of the cultures be apparent Cultures aged $21 \pm 1$ DIV exhibited robust bursting behaviour, therefore the first criterion was met by choosing cultures of this age where the majority of the channels showed regular burst activity. The latter criterion was satisfied by ensuring that all recordings were made under electrical and pharmacological control conditions. In total, 24 recordings met these criteria and were studied in this investigation.

\subsection{Burst detection}

Network burst detection was performed using the SIMMUX algorithm [23], which identifies bursts as periods of significantly heightened spiking frequency that spanned multiple channels. Overlapping bursts were merged to give a sequence of bursts $\beta_{r, b}$ for each recording $r \in[1 \ldots 24]$, such that $b \in\left[1 \ldots \eta_{r}\right]$, where $\eta_{r}$ is the number of bursts detected in recording $r$. For each burst, the duration was defined as the time between the first spike and the last spike and the size was defined as the number of spikes in the burst, across all channels. The inter-burst interval (IBI) was defined as the time between the last spike of one burst and the first spike of the subsequent burst. For each burst, channels that contributed more than $0.15 \mathrm{~N}$ spikes to the burst were said to be active, where $N$ was the number of spikes on the channel that contributed the most.

\subsubsection{Burst classification}

The burst size distributions from recordings were classified into three categories: fixed and variable distributions (unimodal with low and high variance, respectively) and bimodal distributions (containing two distinct classes of burst sizes) [7]. The classification was done using the technique described in [7], by first dividing the burst size distribution into three segments (classifying small, medium, and large bursts). First a burst size, $N$, was chosen as the third largest burst in the sequence, then bursts were classified such that small $<0.25 \mathrm{~N} \leq$ medium $<$ $0.75 N \leq$ large. Then the burst distributions were defined as shown in equation 1. Examples of the three burst distribution classes and their frequencies within the data set are shown in Figure 1.

$$
\begin{aligned}
\text { Fixed } & \equiv \mid \text { large }|>| \text { medium }|\geq| \text { small } \mid \\
\text { Variable } & \equiv \mid \text { medium }|\geq| \text { large } \mid \\
\text { Bimodal } & \equiv \mid \text { large }|>| \text { medium }|<| \text { small } \mid
\end{aligned}
$$

, where $|\cdot|$ denotes the number of bursts in a segment.

\subsubsection{Temporal burst analysis}

Before considering the spatio-temporal properties of functional connectivity in these bursts, some analysis was performed to assess the linear dependencies between the burst properties. This analysis involved performing auto- and cross-correlations between the burst durations, sizes, and preceding IBls to judge how these properties interrelate. 


\subsection{Correlations and significance}

A number of tests for linear dependence between variables were performed in this study using correlation. These correlations were performed by finding the Pearson's Coefficient of Correlation between two sequences of values at 0 or several lags. At each lag step, significance was assessed using Student's t-test, $\alpha=0.05$, unless stated otherwise.

\subsection{Synchrony analysis}

For the two spike trains, $s_{i}$ and $s_{j}$, simultaneously recorded from channels, $c_{i}$ and $c_{j}$, the synchrony between them was defined by $\sigma\left(s_{i}, s_{j}\right)$. There are many ways to assess and define synchronous spike activity $[16,17]$, but for the purpose of this study, synchrony between two spike trains was defined as reoccurring coincident spike patterns. Two parallel techniques were chosen to evaluate $\sigma$ to ensure that the results would not be overly biased by the chosen technique.

The first, denoted $\sigma_{D}$, is related to lagged correlation and mutual information [24] and requires that the spike trains be discretised into binary vectors. While this technique is more conventional, it has a number of known disadvantages $[24,25]$. The second technique, denoted $\sigma_{S}$, is related more to the concept of phase synchrony [17] and requires that non-binary instantaneous phase vectors be created from each spike train [28]. This technique is faster, time-scale adaptive, but less commonly used than the first. Since bursts tend to begin with extremely high spike frequency and then fade out, the time-scale adaptive property of this technique might be helpful. In both cases, $\sigma$ was normalized relative to the expected synchrony from two independent spike trains with the same spiking frequencies as $s_{i}$ and $s_{j}$. Both techniques are commutative and result in symmetric similarity matrices.

These similarity matrices were then thresholded to produce unweighted and undirected functional connectivity networks (FCNs), $G_{r, b}^{D}$ and $G_{r, b}^{S}$, respectively, where $b$ is the index of the burst in recording $r$. The FCNs are defined on a constant set of nodes that represent the electrodes of the MEA. Edges between the nodes represent significant synchrony.

\subsubsection{Binned correlation, $\sigma_{D}$}

Taking inspiration from the Unitary Events method discussed in [24], the spike trains were first discretised into binary vectors of $1 \mathrm{~ms}$ bins, $s_{i}^{*}$ and $s_{j}^{*}$ of length $n$, where a bin had a value of 1 when at least one spike occurred during that bin and 0 otherwise. Then, $\sigma_{D}\left(s_{i}, s_{j}\right)=\sigma_{D}^{*}\left(s_{i}^{*}, s_{j}^{*}\right)$ (equation 2) gave the similarity value between these two vectors. A maximum lag of 14 ms was chosen to reflect the likely maximum time window in which to observe a monosynaptic interaction across an MEA. Since the vectors are much longer than the number of lag steps used $(\approx 700$ vs 14$)$, the reduction in temporal overlap from lags is negligible. Note that $\sigma_{D}^{*}$ is normalised such that $\sigma_{D}^{*}>1$ if there are more synchronous events than one might expect from two independent spike trains with spiking frequencies of $s_{i}$ and $s_{j}$, and $\sigma_{D}^{*} \leq 1$ otherwise.

$$
\sigma_{D}^{*}\left(s_{i}^{*}, s_{j}^{*}\right)=\max _{\ell \in[-14,14]} \frac{n\left(s_{i}^{*}(t-\ell) \cdot s_{j}^{*}(t)\right)}{\sum_{t} s_{i}^{*}(t) \sum_{t} s_{j}^{*}(t)}
$$

\subsubsection{Binless SPIKE distance, $\sigma_{S}$}

The binless technique used here was the SPIKE distance, defined in [28], where SPIKE $=0$ when two spike trains are identical and SPIKE $\rightarrow 1$ as the spike trains become progressively desynchronised. To mitigate the effect of frequency, an empirical distribution was created from the SPIKE value of surrogate spike trains, generated using dithering (100 surrogates, 5 ms jitter window) [24]. A p-value,
$p_{S}\left(s_{i}, s_{j}\right)$, was found from the empirical distribution to indicate the significance of the SPIKE distance. Thus, $\sigma_{S}\left(s_{i}, s_{j}\right)=1-p_{S}\left(s_{i}, s_{j}\right)$ such that $\sigma_{S} \rightarrow 1$ when two spike trains become more synchronised than expected by chance.

\subsubsection{Thresholding}

Once $\sigma$ values were found for each pair of channels in a burst, a threshold was chosen to convert the similarity matrices into FCNs. To ensure that all active nodes had neighbours, equation 3 was used to define a uniform threshold, $T$, such that an edge was placed between nodes and $j$ in a network if $\sigma\left(s_{i}, s_{j}\right)>=T$.

$$
T=\min _{i}\left[\max _{j} \sigma\left(s_{i}, s_{j}\right)\right]
$$

\subsection{Complex network models}

Each recording produced a sequence of FCNs, $G_{r, b}$, associated with the bursts found in that recording. These FCNs represent a stochastic series of undirected and unweighted complex networks on a fixed set of $v$ nodes. Thus, in any burst, an FCN, $G_{r, b}$, is a network randomly chosen from the space of all possible networks on $v$ nodes, where significant synchrony between nodes $i$ and $j \in[1 \ldots v]$ during burst $b$ of recording $r$ is denoted $e_{i j}(r, b)=1$ and 0 otherwise. As $v$ increases, this state space scales as $2^{v^{2}}$, making the problem quickly intractable. By understanding the precise dependencies in the system, a concise model might be produced that adequately captures the dynamics.

To investigate these spatio-temporal dependencies in the FCNs, three network models were considered. The first two models were homogeneous and heterogeneous Erdös-Renyi random graph models, denoted HomER and HetER, respectively. The HomER model assumes that edges appear in an FCN independently of each other, their place in the network, and of their own pasts. The HetER model maintained the temporal and inter-edge independence, but considers that edges may appear more frequently between some nodes than between others. For each recording, $r$, the HomER model's single parameter, $p_{r}=\mathbf{P}\left(e_{i j}(r, b)=1\right)$, was fit according to equation 4 , and the HetER model's $v^{2}$ parameters, $p_{r i j}=\mathrm{P}\left(e_{i j}(r, b)=1\right)$, were fit according to equation 5 .

$$
\begin{gathered}
p_{r}=\frac{1}{\eta_{r} v^{2}} \sum_{b=1}^{\eta_{r}} \sum_{i=1}^{v} \sum_{j=1}^{v} e_{i, j}(r, b) \\
p_{r i j}=\frac{1}{\eta_{r}} \sum_{b=1}^{\eta_{r}} e_{i, j}(r, b)
\end{gathered}
$$

The third model, denoted the Independent Birth-Death model (IndBD), was based on a model presented by Grindrod and Higham [26], in which edges are dependent on their pasts but remain independent of each other. In this model, an edge is a binary Markovian birthdeath process $\left(\mathbf{P}\left(e_{i j}(r, b) \mid e_{i j}(r, 1), e_{i j}(r, 2), \ldots, e_{i j}(r, b-1)\right)=\right.$ $\left.\mathrm{P}\left(e_{i j}(r, b) \mid e_{i j}(r, b-1)\right)\right)$, such that it can be born (transition from $0 \rightarrow 1$ ) or die (transition from $1 \rightarrow 0$ ) as the network transitions from $(b-1) \rightarrow b$. Thus the model for each recording is defined by the $2 v^{2}$ parameters defined in equation 6 .

$$
\begin{aligned}
& \alpha_{r i j}=\mathrm{P}\left(e_{i j}(r, b)=1 \mid e_{i j}(r, b-1)=0\right) \\
& \omega_{r i j}=\mathrm{P}\left(e_{i j}(r, b)=0 \mid e_{i j}(r, b-1)=1\right)
\end{aligned}
$$


These birth paramters, $\alpha$, and death parameters, $\omega$, were fit using expectation maximization, as described in [26].

\subsubsection{Model capture performance}

Each model was evaluated to compare how well each captured the dynamics of the data. The evaluation was performed first by considering how well individual edge series were captured, and then by considering how well entire network series were captured.

The capture performance was evaluated by using each fitted model to generate 1000 surrogate networks, whose likelihoods, given the model that created them, were used to create an empirical distribution for that model. Then, the likelihood of each real network sequence was found, given the model which was fitted to it, and compared to the empirical distributions to get a p-value. The p-value for a model indicated the probability that the model could have generated the real sequence and was used to assess the quality of fit. Since all three models have edges that evolve independently, the likelihood of a network is simply the product of the likelihoods of the edges.

\subsection{Network topology analysis}

All three models described in section 2.5 assume that the edges in the FCNs are independent of each other. To evaluate this hypothesis, four topological properties were compared between the real networks and model generated surrogates. Since brain networks regularly have a small-world topology [11], the first three measures evaluated this property: average path length (also called characteristic path) and mean clustering coefficient measured the long and short range efficiency, respectively $[20,27]$, and small-worldness was used to evaluate whether the MCC to APL ratio was greater than might be expected in equivalent random networks [18]. The last was mean node degree, a typical indicator of the edge density of a network [20].

For each FCN and its related surrogates, these four measures were calculated and their distributions across the bursts within each recording were compared.

\section{Results}

\subsection{Network bursts}

Twenty-four electro-physiological recordings taken from rodent cortical neurones cultured on MEAs were analysed and bursts of excitation were identified using the SIMMUX algorithm and their sizes, durations, and inter-burst intervals were identified (see section 2.2). The linear dependencies between these properties were assessed with crosscorrelation. A strong positive correlation was found between the burst size and burst duration, which is unsurprising given that neurones have a minimum post-firing refractory time, limiting their maximum spiking frequency.

The nature of periodicity in the bursting patterns of the cultures was investigated, first by defining burst periods as the duration of a burst combined with its preceding inter-burst interval, and then by scatterplotting and correlating each period duration with the subsequent one (Figure 2). It was found that many variable and bimodal cultures were periodic, where long burst periods tended to follow short ones and viceversa. Fixed distribution cultures showed a more positive linear relationship, but the bursts in these cultures are by definition very similar in size.
Table 1. Model Capture Performance. The percentage of models that fit $(p \in[0.025,0.975])$ their corresponding data sets are shown for each model and each data set.

\begin{tabular}{|l||l|l||l|l|}
\hline \multirow{2}{*}{ Model } & \multicolumn{2}{|c||}{ Mean edge fit } & \multicolumn{2}{c|}{ Mean network fit } \\
\cline { 2 - 5 } & Binless & Binned & Binless & Binned \\
\hline HomER & $46.48 \%$ & $38.64 \%$ & $100.00 \%$ & $100.00 \%$ \\
HetER & $99.58 \%$ & $99.54 \%$ & $100.00 \%$ & $100.00 \%$ \\
IndBD & $99.45 \%$ & $99.78 \%$ & $70.83 \%$ & $83.33 \%$ \\
\hline
\end{tabular}

\subsection{Functional connectivity}

The binned and binless synchrony techniques described in section 2.4 were applied to each burst from each culture and the results were thresholded to yield sequences of functional connectivity networks for each culture. For each network of each sequence, four network properties were calculated: Average Path Length (APL), Mean Clustering Coefficient (MCC), Mean Node Degree (MND), and Small-Worldness (SW); refer to section 2.6 for details. In Figure 4, the topological parameters from binned and binless functional connectivity networks were compared and showing that the two techniques are largely analogous.

\subsubsection{Range dependence}

In 19 of the binless sequences and in 14 of the binned sequences, edges were significantly more likely to appear between a pair of nodes if their corresponding electrodes were closer on the MEA. In a single binned sequence, there was a small but significant positive relationship which can be explained by there being no activity on the central electrodes of the MEA (likely because the cells in the centre had died or lifted off the surface). However, none of these correlations were stronger than $-0.3,14 \%$ were stronger than -0.2 , and around $30 \%$ were not significant at all.

\subsubsection{Topological dependence on burst size}

The effect of burst size on each of the four topological properties from a network was assessed by correlating each property with the size of the burst that generated the network. Scatter-plots of these comparisons are shown in Figure 3 for network sequences produced by each technique.

In most sequences produced by both synchrony techniques, the mean degrees of the networks increased as the bursts grew in size and this had direct consequence for mean clustering. The relationship between average path length and burst size was not quite as clear, though. With increased numbers of edges in the network, one would have expected the APL to decrease, and while it did for the binned variable cultures, the opposite was generally true for all others, especially for bimodal cultures. Scatter-plotting APL versus burst size for bimodal cultures, it was not clear that there was as strong a linear relationship as was suggested by the correlation results.

\subsubsection{Models of functional connectivity}

The three models, HomER, HetER, and IndBD (described in section 2.5), were fitted to the sequence of networks from each culture and were each used to generate 1000 sequences of surrogate networks for each culture. The surrogate network sequences generated from a model were used to represent the space of all likely such sequences. By comparing these surrogate sequences to those derived from the culture recordings, the validity of the hypotheses in the models were tested.

The first test for model fit was done by assessing the probability that 

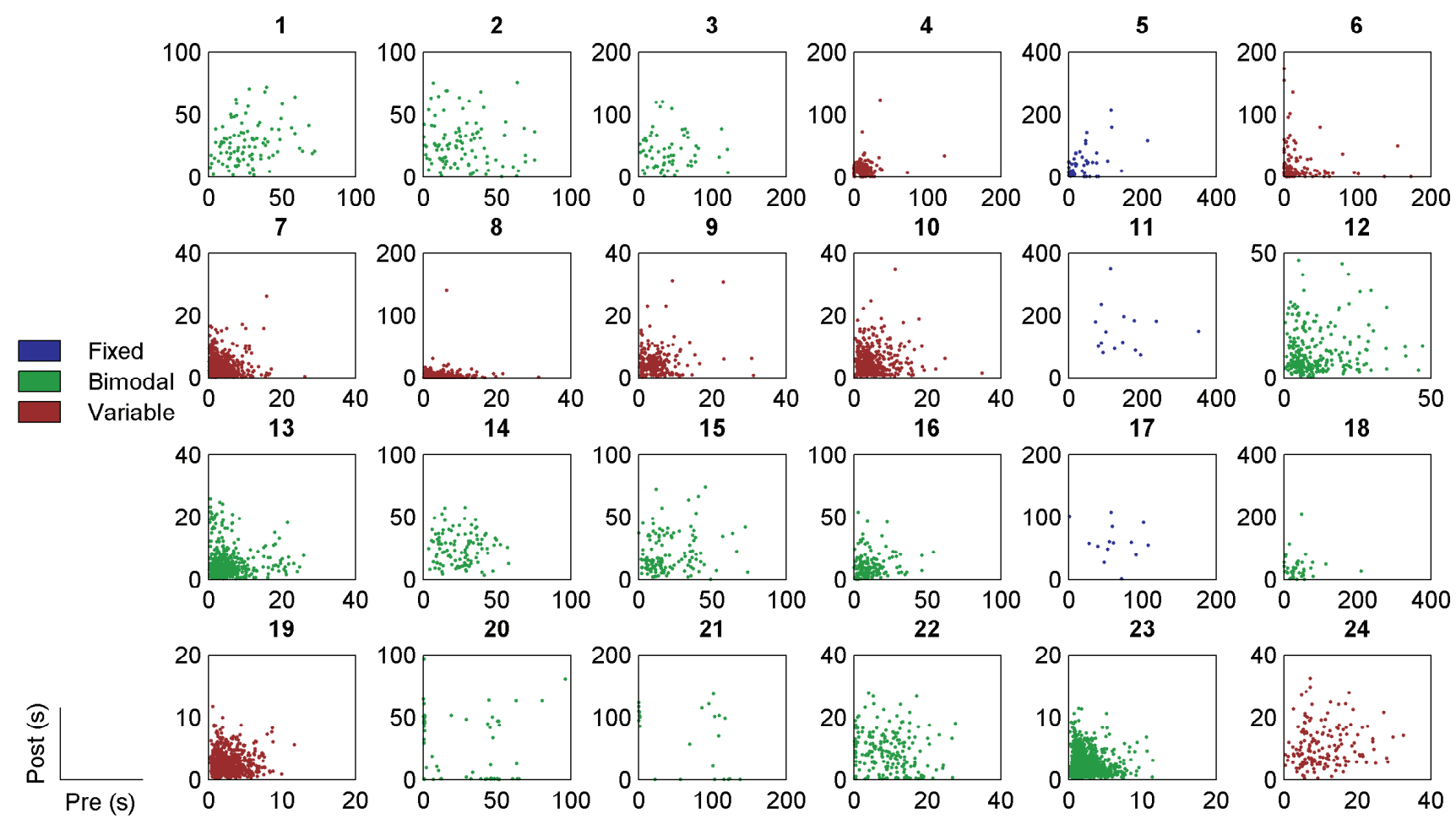

Figure 2. Temporal burst period dependence. Burst period lengths from each the 24 recordings are scatter-plotted against the subsequent burst period length. The burst period is the duration of a burst combined with the preceding period of quiescence (the inter-burst interval). For most cultures, longer periods are generally followed by shorter ones and vice-versa, though this is not universal.

each model could have produced the real network sequence to which it was fitted. This was done, as described in section 2.5.1, for the time evolution of each edge through a sequence of networks and for the entire network sequences themselves. The percentage of edge and network models where the p-values were within $95 \%$ of the empirical distribution are shown in table 1. The HetER and IndBD models fit the time-evolving edge series best, but the HetER and HomER models fit the time-evolving network series best.

To assess how each model captured the topological properties of the data, these properties were calculated from the surrogate networks and the results were compared. It was found that while MND was captured in mean, but not in variance, APL was generally overestimated by all three models and MCC was generally underestimated (Table 1). Since Small-Worldness is defined as having higher MCC and lower APL than equivalent random networks, it was not surprising to see SW also generally underestimated.

\section{Discussion}

To assess the spatio-temporal dependencies in both excitation and functional connectivity within network bursts, correlations were performed between burst properties, functional connectivity networks were produced, and the properties of these networks were analysed. The burst property correlations found that most variable and bimodally distributed cultures tend to a degree of periodicity, in which short burst periods follow long ones, and vice-versa

Once functional connectivity networks (FCNs) were constructed from the bursts, further spatial and temporal dependencies were investigated between the synchronous links. In many cultures, significant range dependence was found, suggesting that synchronous links were more likely to be found between electrodes that are located close together on the surface of the MEA. This is not surprising, considering that proximal electrodes may detect much of the same local field potential and that neighbouring neurones might be more likely to be synaptically connected. However these correlations were inconsistent and minor suggesting that the distance between a pair of electrodes is not a major factor in predicting the likelihood of a functional connection between them.

Topological properties were calculated from the networks and these were compared to the sizes of the bursts that had produced them. It was found that in variable and bimodal cultures, larger bursts tended to have larger minimum clustering coefficients and larger minimum node degrees, though the values do not linearly follow the burst size. In bigger bursts, more channels are active in the burst, increasing the maximum number of possible functional links. Thus, it is not surprising to observe that larger bursts might produce more edges (as reflected by the degree), however because these additional edges also come with additional nodes, the degree, clustering, or path length do not necessarily follow linearly.

Spatiotemporal relationships between edges were investigated through the use of three models of edge appearance in functional networks. The first model, HomER, hypothesised that edge might appear com- 
APL

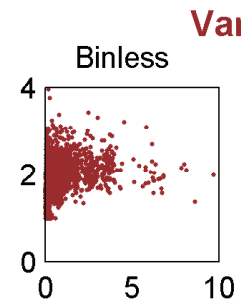

Variable
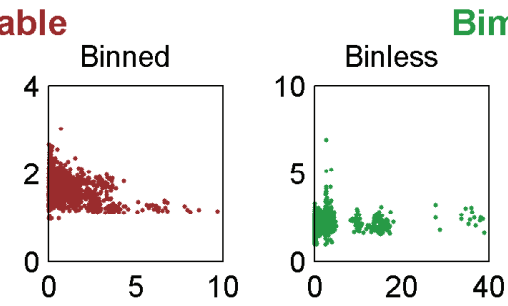

Bimodal
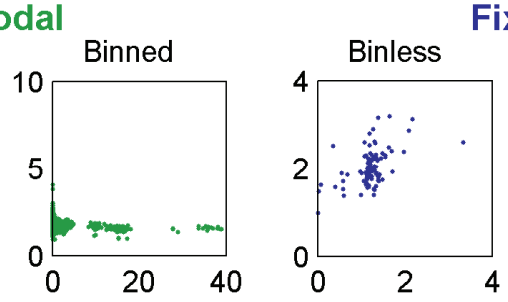

Fixed
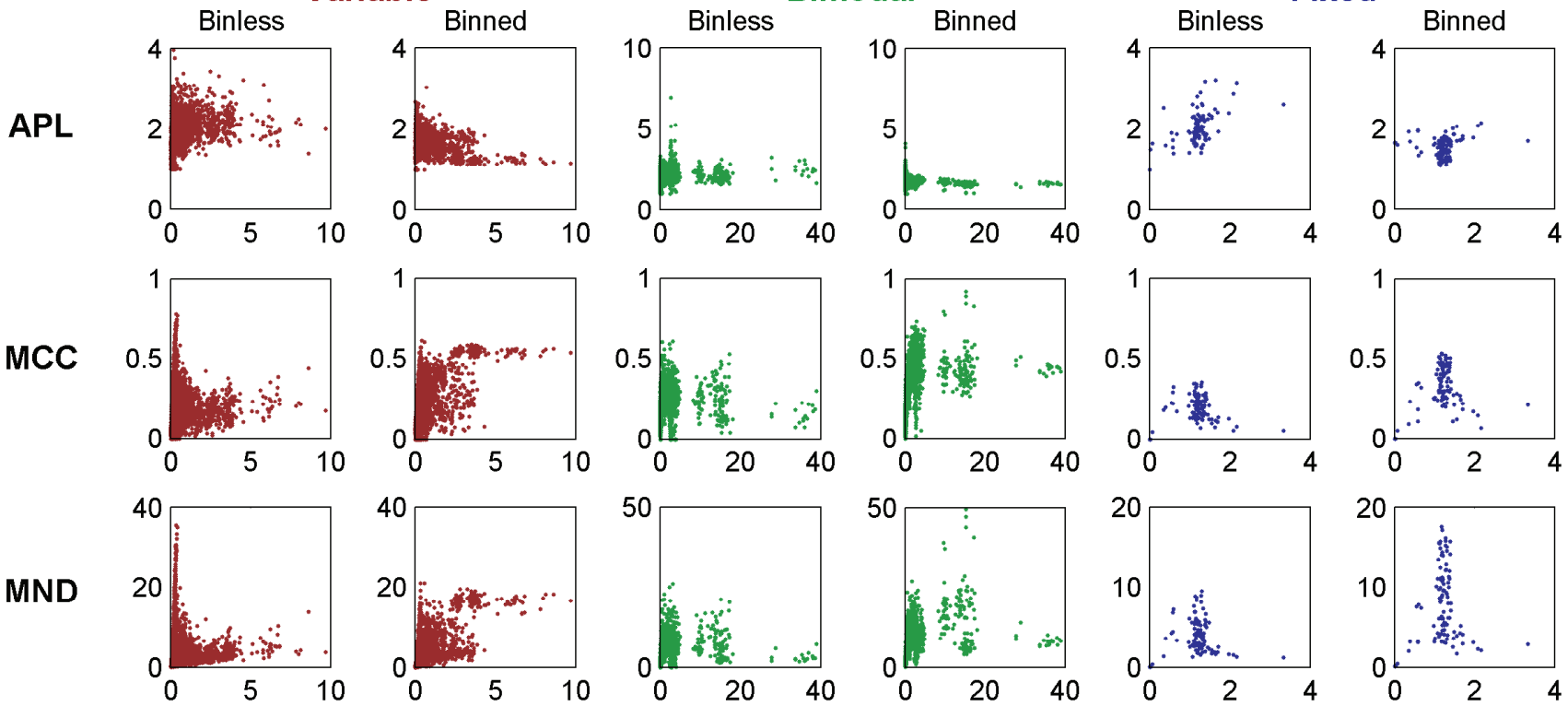

MND
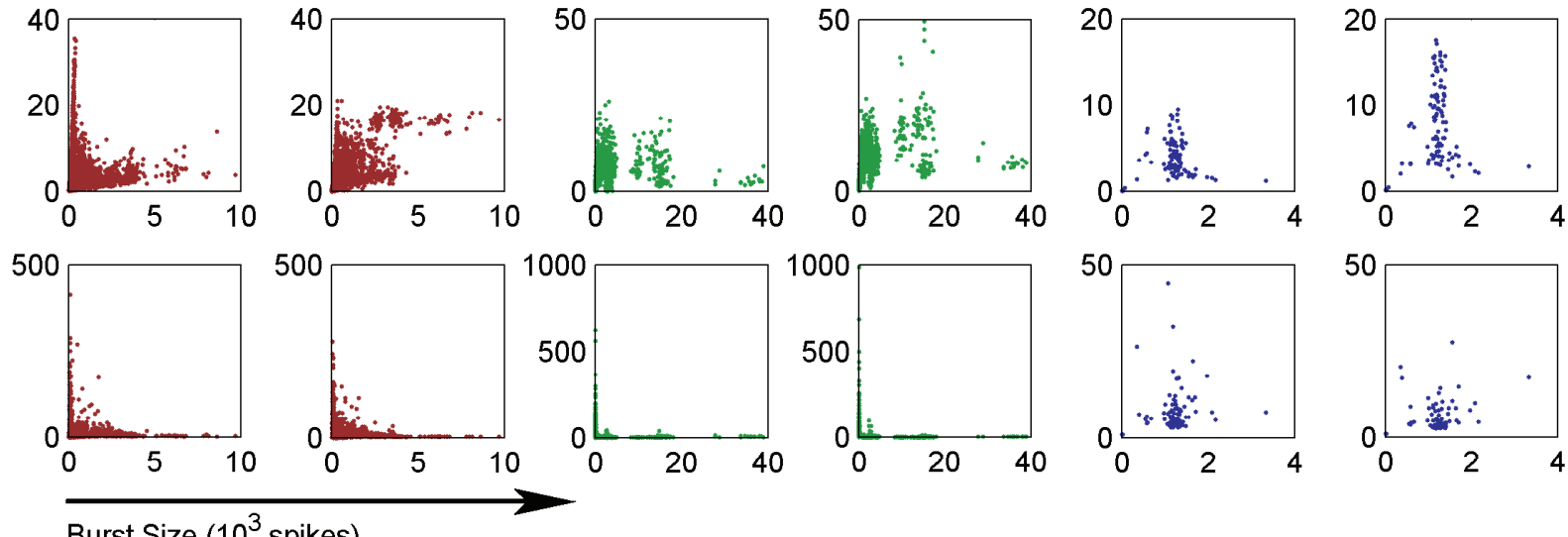

Burst Size $\left(10^{3}\right.$ spikes $)$

Figure 3. Topological dependence on burst size in network sequences. Each of the four topological properties (Average Path Length, Mean Clustering Coefficient, Mean Node Degree, and Small-Worldness) from each of the three classes (variable, bimodal, and fixed) and constructed with each synchrony technique (Binless and Binned) are shown scatter-plotted against burst size, measured in $10^{3}$ spikes.
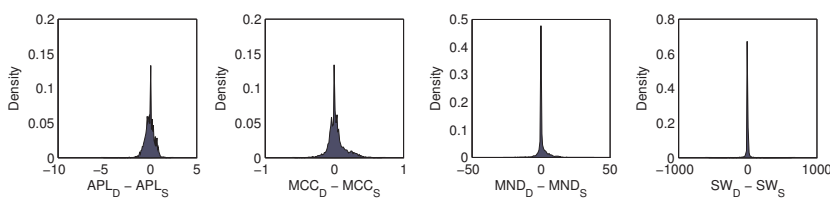

Figure 4. Comparison of topological properties between Binned networks and Binless networks. The synchrony in the Binned networks, D, was assessed using a binned covariance measure, while the synchrony assessed using a binned covariance measure, while the synchrony
in the Binless networks, S, was assessed using the binless SPIKE distance measure. The dramatic peaks at $O$ indicate that the two techniques produce networks with very similar topologies, with the binned technique producing slightly more edges, as indicated by the right weighted MND and MCC, and left-weighted APL.

pletely at random between any nodes, independent of each other or their pasts. For capturing individual edge progressions through sequences of networks, this model was a poor choice, only accurately capturing the dynamics of $46.48 \%$ and $38.64 \%$ of the edge individual sequences found in the data, for Binless and Binned networks respec- tively. However, this model quite accurately captured the network progression dynamics when assuming that all edges were independent. The second model, HetER, hypothesised that each pair of nodes might have a specific probability of being joined by a functional link in a given network, but maintained that these links would be independent of each other and their pasts. This model very accurately captured both the edge and network progression dynamics.

The final model, IndBD, added to the HetER hypothesis that the appearance of an edge between a pair of nodes may also be dependent on whether those nodes were functionally linked in the previous burst. Since the edge appearance dynamics were modelled with ergodic Markov chains, the steady-state behaviour closely resembled that of the independent identically distributed edge dynamics of the HetER model. However, while this model captured the edge dynamics very accurately, only $70 \%$ and $83 \%$ of the network sequences' dynamics were captured, for Binless and Binned networks, respectively. This suggests that the functional network observed during a burst has little dependence on the network observed during the previous burst.

The three models used in this study assume that the edge dynamics in functional networks are independent from each other. The validity of this assumption was tested by observing the general topological properties of surrogate networks generated by these models and 
Binless

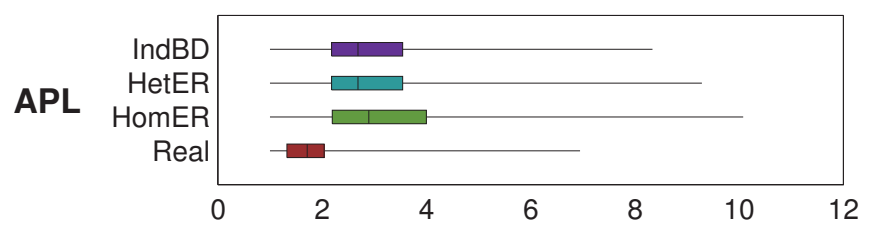

MCC

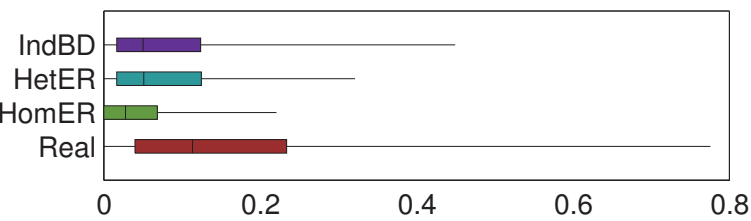

MND

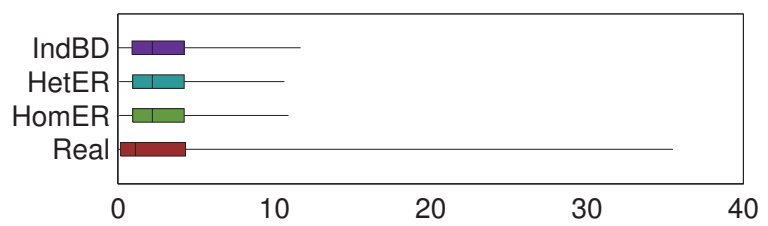

SW

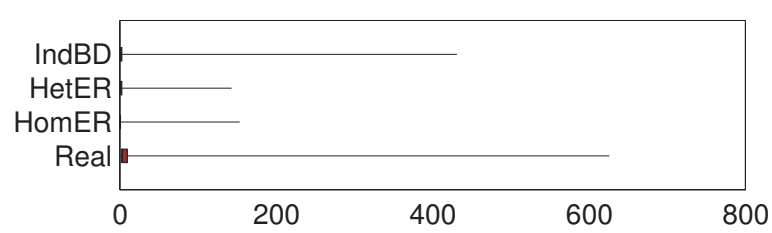

Binned
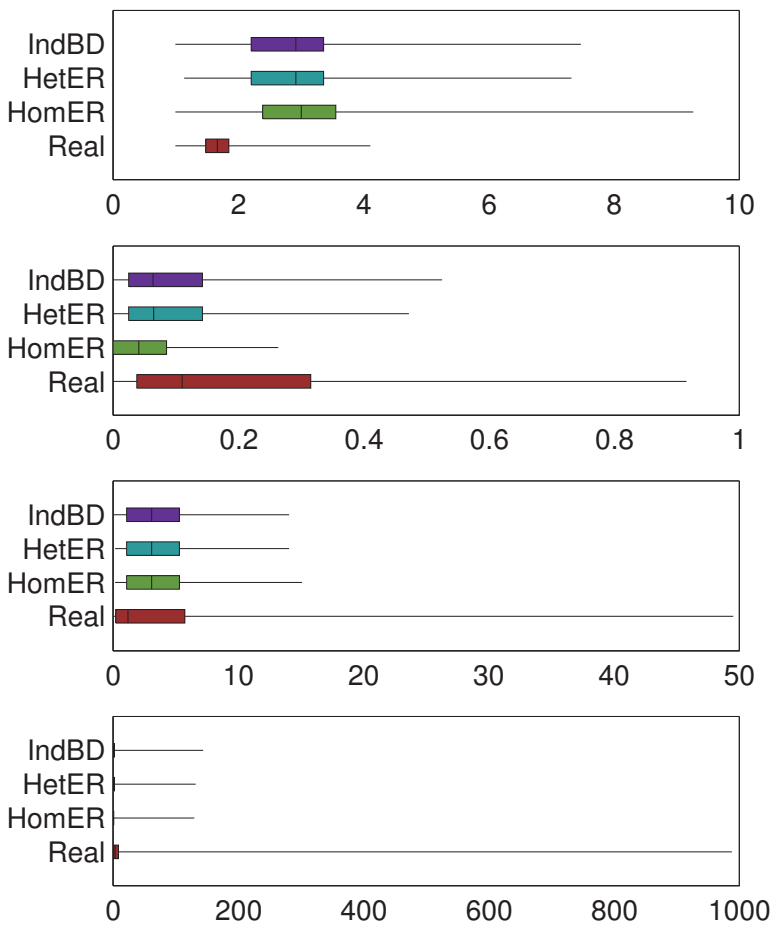

Figure 5. Topological properties of networks produced from real data sets and the models fitted to them. Each subplot shows the aggregate distributions of one network property from networks generated using one of the two synchrony techniques (denoted real) and the surrogate network generated from the three models fit to those networks. Regardless of synchrony technique. Average Path Length is generally overestimated by the models and Mean Clustering Coefficient is generally underestimated. The models tend to reproduce the average mean node degree of the data, but not the variance. Since APL and MCC are poorly captured, Small-Worldness is correspondingly underestimated.

comparing them to analogous properties from the real data. In both Binless and Binned networks, the average path length was generally over-estimated by the models, and the mean clustering coefficient was generally under-estimated. The models tended to produce an average mean node degree similar to the data, but the data exhibited more than triple the variation. The lower MCC and higher APL of the surrogate networks shows that none of the models capture the small-world properties of the biological networks; properties that are thought to be crucial to information passing and storage [11].

By assessing synchrony by two different techniques, it was found that while the Binned technique tended to produce slightly denser networks, the specific definition of functional connectivity did not drastically affect the small-world properties of the networks.

\section{Conclusion}

This study used a variety of techniques to investigate the spatial and temporal dependencies of evolving functional connectivity in cultures of cortical neurones. Understanding these dependencies will provide the insight necessary to construct models that can accurately capture the dynamics of bursts of activity in neuronal networks. Such models would be useful for classifying burst dynamics, gaining insight into neuronal network structure, and planning interventions to prevent or alter the nature of these bursts, allowing further understanding of the computational capabilities of biological neuronal networks and perhaps enabling their use as suitable robotic controllers [5]. While the present study focuses on randomly arranged neuronal networks in culture, these findings might generalise to the highly structured networks found in the whole brain, where network bursts are either formative or pathological [8], and interventions might be used to repair damage or pre-empt seizures.

This study found that many cultures tend to exhibit periodic sequences of longer bursts followed by shorter bursts and vice-versa. It was also found that the functional networks within these bursts do not depend on the preceding bursts, but do have strong small-world topologies that are not captured by models that lack inter-edge dependencies. This means that not all functional pathways are activated in every burst, nor are pathways regularly activated in subsequent bursts. However, the presence of spatial dependencies would indicate that when a pathway between two nodes is activated, it is activated along with a regular set of other pathways. This might point to the presence of discrete subnetworks or consistent information flow pathways within the neuronal network, as are found in memory recall, discrimination, and decision making in whole brain structures.

Random network models that aim to make use of these findings would need to consider the inter-edge dependencies and the resultant smallworld characteristics as well as the variability of burst durations and inter-burst intervals. While the Watts-Strogatz model for randomly gen- 
erating small-world networks [18] or the configuration model for generating networks with proscribed degree distributions [20] might be reasonable places to start, neither of these models can capture the interedge dependencies or the variability seen in the data. Instead, it would likely be better to search for a model inspired by the underlying biological system, and perhaps one that uses finer-grained and more regular time-steps than those investigated here. Such a model, using the past interplay between node dynamics to construct functional connections at any given step has been recently shown to be promising [29]. Another possible direction might be to track a resource, such as an abstract 'cellular energy' which might encompass a variety of intra-cellular properties (eg. number of available vesicles), and use this resource to regulate the frequency and duration of bursting events. Also, once a suitable model has be found to capture the dynamics in the data, such a model would be limited by the manageability of its inverse solution. Supposing that these challenges can be overcome, these models may provide a key to improving clinical neurological treatments, understanding neuronal network function, and building the next generation of interfaces between biological tissue and robotic systems.

\section{Acknowledgement}

The authors would like to thank Prof S. Potter at the Georgia Inst. of Technology for generously providing the set of neuronal recordings used for this study.

\section{References}

[1] E. Maeda, H. P. Robinson, and A. Kawana The mechanisms of generation and propagation of synchronized bursting in developing networks of cortical neurons, J. Neurosci., 15(10), (1995), 6834-45

[2] Y. Jimbo, T. Tateno, and H. P. C. Robinson, Simultaneous induction of pathway-specific potentiation and depression in networks of cortical neurons, Biophysical Journal, 76, (1999), 670-678

[3] G. Shahaf and S. Marom, Learning in networks of cortical neurons, J. Neurosci., 21(22), (2001), 8782

[4] J. van Pelt, P. S. Wolters, Michael A. Corner, W. L. C. Rutten, and Ger J. A. Ramakers, Long-term characterization of firing dynamics of spontaneous bursts in cultured neural networks, IEEE Trans. Bio-med. Eng., 51(11), (2004), 2051-62

[5] T.B. DeMarse, D.A. Wagenaar, A.W. Blau, and S.M. Potter, The neurally controlled animat: biological brains acting with simulated bodies, Autonomous Robots, 11(3), (2001), 305-310

[6] M.W. Hammond, S. Marshall, J. Downes, D. Xydas, S.J. Nasuto, V.M. Becerra, K. Warwick, and B.J. Whalley, Robust methodology for the study of cultured neuronal networks on MEAs, BadenWurttemberg GmbH, Stuttgart, 2008

[7] D. A. Wagenaar, J. Pine, and S. M. Potter, An extremely rich repertoire of bursting patterns during the development of cortical cultures, BMC Neuroscience, 7:11, (2006)

[8] J. van Pelt, I. Vajda, P. S. Wolters, M. A. Corner, and Ger J. A. Ramakers, Dynamics and plasticity in developing neuronal networks in vitro, Prog. Brain Res., 147, (2005), 173-88

[9] D. A. Wagenaar, J. Pine, and S. M. Potter, Searching for plasticity in dissociated cortical cultures on multi-electrode arrays, Journal of Negative Results in Biomedicine, 5:16, (2006)

[10] M. Chiappalone, A. Novellino, I. Vaida, A. Vato, S. Martinoia, and J. Van Pelt, Burst detection algorithms for the analysis of spatio- temporal patterns in cortical networks of neurons, Neurocomputing, 65-66, (2005), 653-662

[11] E. Bullmore and O. Sporns, Complex brain networks: graph theoretical analysis of structural and functional systems, Nature Reviews Neuroscience, 10(3), (2009), 186-98

[12] C. J. Honey, R. Kötter, M. Breakspear, and O. Sporns, Network structure of cerebral cortex shapes functional connectivity on multiple time scales, Proc. Nat. Acad. Sci. USA, 104(24), (2007), 10240-5

[13] R. Madhavan, Z. C. Chao, and S. M. Potter, Plasticity of recurring spatiotemporal activity patterns in cortical networks, Physical Biology, 4(3), (2007), 181-193

[14] M. Kaiser, Hierarchy and dynamics of neural networks, Frontiers in Neuroinformatics, 4(August), 10-12, 2010

[15] A. Minerbi, R. Kahana, L. Goldfeld, M. Kaufman, S. Marom, and N. E. Ziv, Long-term relationships between synaptic tenacity, synaptic remodeling, and network activity, PLoS Biology, 7(6), (2009), e1000136

[16] J. D. Victor, Spike train metrics, Current Opinion in Neurobiology, 15(5), (2005), 585-92

[17] E. Pereda, Q. R. Quiroga, and J. Bhattacharya, Nonlinear multivariate analysis of neurophysiological signals, Progress in Neurobiology, 77(1-2), (2005), 1-37

[18] D. J. Watts and S. H. Strogatz, Collective dynamics of 'small-world' networks, Nature, 393, (1998), 440-442

[19] P. Borgnat, E. Fleury, J. L. Guillaume, C. Magnien, C. Robardet, and A. Scherrer, Evolving networks, In Françoise FogelmanSoulié, Domenico Perrotta, Jakub Piskorski, and Ralf Steinberg editors, Proceedings of NATO Advanced Study Institute on Mining Massive Data Sets for Security, 198-204. IOS Press, 2008

[20] S. Boccaletti, V. Latora, Y. Moreno, M. Chavez, and D. Hwang, Complex networks: Structure and dynamics, Physics Reports, 424(4-5), (2006), 175-308

[21] M. C. Spencer, D. Xydas, J. H. Downes, M. W. Hammond, V. M. Becerra, K. Warwick, B. J. Whalley, and S. J. Nasuto, Investigation of spatio-temporal dependencies in neuronal functional connectivity, In: 9th IEEE Int. Conf. Cyber. Intel. Sys., 2010

[22] U. Egert, D. Heck, and A. Aertsen, Two-dimensional monitoring of spiking networks in acute brain slices, Experimental Brain Research, 142(2), (2002), 268-74

[23] D. A. Wagenaar and T. B. DeMarse, Meabench: A toolset for multi-electrode data acquisition and on-line analysis, Proc. 2nd Int. IEEE EMBS Conf. Neural Eng., 2005

[24] S. Grün, Data-driven significance estimation for precise spike correlation, Journal of Neurophysiology, 101(3), (2009), 1126-40

[25] A. R. C. Paiva, I. Park, and J. C. Príncipe, A comparison of binless spike train measures, Neural Computing \& Applications, 19(3) (2010), 405-419

[26] P. Grindrod and D. J. Higham, Evolving graphs: dynamical models, inverse problems and propagation, Proc. Roy. Soc. A 466(2115), (2009), 753-770

[27] R. Albert and A. L. Barabási, Statistical mechanics of complex networks, Reviews of Modern Physics, 74(1), (2002), 47-97

[28] T. Kreuz, D. Chicharro, M. Greschner, and R. G. Andrzejak, Timeresolved and time-scale adaptive measures of spike train synchrony, J. Neurosci. Methods, 195(1), (2010), 92-106

[29] M. C. Spencer, J. H. Downes, D. Xydas, M. W. Hammond, V. M. Becerra, K. Warwick, B. J. Whalley, and S. J. Nasuto, Multi-scale evolving complex network model of functional connectivity in neuronal cultures, IEEE Trans. Bio-med. Eng., (2011), 1-5 